\title{
Desempenho agronômico de genótipos de amendoim nas condições de Campo Verde-MT
}

Submetido - 07 jul. $2020 \quad$ Aprovado - 10 set. $2020 \quad$ Publicado - 14 out. 2020
dol http://dx.doi.org/10.17648/sas.v1i1.9

Maxuel Fellipe Nunes Xavier (D)

Discente de Agronomia do IFMT Campus São Vicente - Centro de Referência de Campo Verde, Campo Verde, MT, maxuelfellipe90@gmail.com.

Gustavo Costa Meinke (D)

Discente de Agronomia do IFMT Campus São Vicente - Centro de Referência de Campo Verde, Campo Verde, MT, gustavomeinke@hotmail.com.

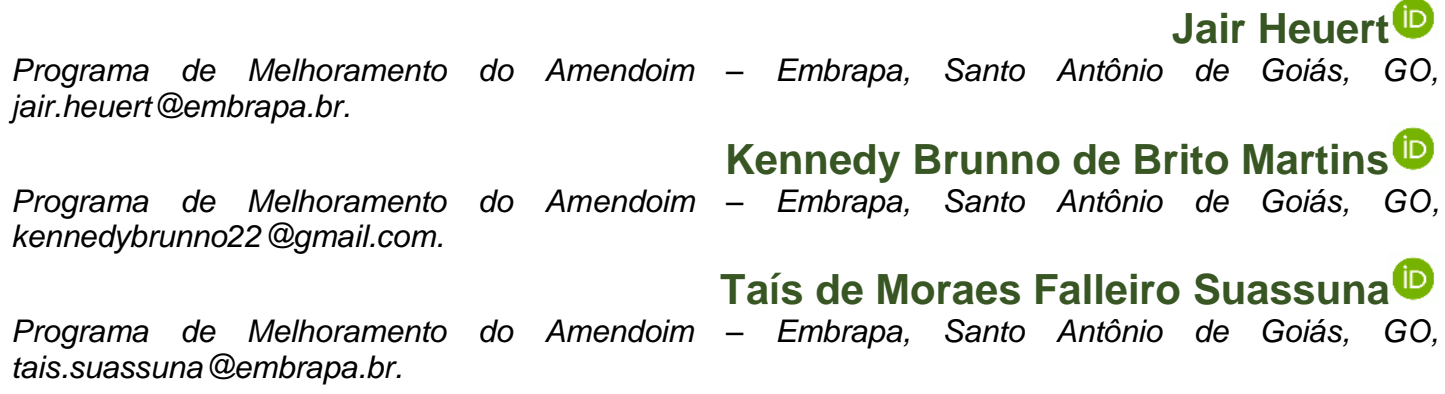

\section{RESUMO}

O presente trabalho teve como objetivo avaliar o desempenho agronômico de genótipos de amendoim: três cultivares e duas linhagens. $O$ experimento foi desenvolvido área experimental do Instituto Federal de Educação, Ciência e Tecnologia de Mato Grosso - Campus São Vicente, Centro de Referência de Campo Verde, localizado no município de Campo Verde - MT, no ano agrícola 2019/20. Os tratamentos constam de cinco genótipos de amendoim rasteiro, três cultivares: BRS 421 OL, BRS 423 OL, BRS 425 OL e duas linhagens avançadas: 17-1253 OL e 18-1952 OL, desenvolvidas pelo Programa de Melhoramento do Amendoim da Embrapa. $O$ delineamento experimental foi em blocos casualizados, com quatro repetições. As parcelas foram compostas por duas linhas de três metros de comprimento, espaçamento ente linhas de $0,90 \mathrm{~m}$, intervalo entre parcelas de dois metros e parcela útil $5,4 \mathrm{~m}^{2}$. Foram avaliados severidade de mancha preta, massa de 100 grãos e produtividade de vagens. Todas as cultivares apresentaram as menores notas de severidade de mancha preta. Os genótipos BRS $425 O L$, BRS 421 OL, 17-1253 OL e BRS 423 OL foram os mais produtivos. Constata-se nesta safra resultados de adaptação agronômica das cultivares BRS 421 OL, BRS 423 OL e BRS 425 OL nas condições edafoclimáticas presentes durante o experimento.

Palavras-chave: Arachis hypogaea L.; Programa de Melhoramento; Amendoim em Mato Grosso.

\section{Agronomic performance of peanut genotypes in Campo Verde-MT conditions}

\begin{abstract}
The present work aimed to evaluate the agronomic performance of peanut genotypes: three cultivars and two strains. The experiment was developed in the experimental area of the Federal Institute of Education, Science and Technology of Mato Grosso - Campus São Vicente, Campo Verde Reference Center, located in the municipality of Campo Verde - MT, in the agricultural
\end{abstract}


year 2019/20. The treatments consist of five groundnut peanut genotypes, three cultivars: BRS $421 O L, B R S 423 O L, B R S 425 O L$ and two advanced strains: 17-1253 OL and 18-1952 OL, developed by Embrapa's Peanut Breeding Program. The experimental design was in randomized blocks, with four replications. The plots were composed by two lines of three meters in length, spacing between lines of $0.90 \mathrm{~m}$, interval between plots of two meters and a useful plot of $5.4 \mathrm{~m}^{2}$. Severity of black spot, mass of 100 grains and pod yield were evaluated. All cultivars had the lowest severity of black spot. The genotypes BRS 425 OL, BRS 421 OL, 17$1253 \mathrm{OL}$ and BRS $423 \mathrm{OL}$ were the most productive. This crop shows results of agronomic adaptation of cultivars BRS 421 OL, BRS $423 \mathrm{OL}$ and BRS $425 \mathrm{OL}$ in the edaphoclimatic conditions present during the experiment.

Keywords: Arachis hypogaea L.; Improvement Program; Peanut in Mato Grosso.

\title{
Rendimiento agronómico de genotipos de maní en condiciones de Campo Verde-MT
}

\author{
RESUMEN
}

\begin{abstract}
El presente trabajo tuvo como objetivo evaluar el rendimiento agronómico de los genotipos de maní: tres cultivares y dos cepas. El experimento se desarrolló en el área experimental del Instituto Federal de Educación, Ciencia y Tecnología de Mato Grosso - Campus São Vicente, Centro de Referencia Campo Verde, ubicado en el municipio de Campo Verde - MT, en el año agrícola 2019/20. Los tratamientos consisten en cinco genotipos de maní y maní, tres cultivares: BRS 421 OL, BRS 423 OL, BRS 425 OL y dos cepas avanzadas: 17-1253 OL y 18-1952 $O L$, desarrolladas por el Programa de Mejoramiento de Maní de Embrapa. El diseño experimental fue en bloques al azar, con cuatro repeticiones. Las parcelas estaban compuestas por dos líneas de tres metros de longitud, espaciado entre líneas de $0.90 \mathrm{~m}$, intervalo entre parcelas de dos metros y una parcela útil de $5.4 \mathrm{~m}^{2}$. Se evaluaron la gravedad de la mancha negra, la masa de 100 granos y el rendimiento de la vaina. Todos los cultivares tuvieron la menor severidad de la mancha negra. Los genotipos BRS 425 OL, BRS 421 OL, 17-1253 OL y BRS 423 OL fueron los más productivos. Este cultivo muestra los resultados de la adaptación agronómica de los cultivares BRS 421 OL, BRS 423 OL y BRS 425 OL en las condiciones edafoclimáticas presentes durante el experimento.
\end{abstract}

Palabras clave: Arachis hypogaea L.; Población; Productividad.

\section{Introdução}

O amendoim (Arachis hypogaea L.) é uma dicotiledônea, pertencente à família das Leguminosas, subfamília Faboideae e gênero Arachis (JUDD et al., 1999). É originária da América do Sul e já era cultivada pelas populações indígenas muito antes da chegada dos europeus no final do século XV. O gênero Arachis compreende cerca de 80 espécies descritas, distribuídas em uma grande variedade de ambientes, desde as regiões costeiras do Brasil e Uruguai até altitudes de $1.450 \mathrm{~m}$ na região dos Andes ao noroeste da Argentina (BERTIOLI et al., 2011).

Conforme a estimativa de área plantada de amendoim primeira safra no Brasil, houve um incremento de 7,4\% da safra 2019/20 (150,2 mil hectares) em relação à safra 2018/19 (139,8 mil hectares). Sobre a produção 
de grãos, estima-se 532,3 mil toneladas colhidas, apresentando um acréscimo de $26,1 \%$ comparado a safra anterior (422,2 mil toneladas) (CONAB, 2020).

A Empresa Brasileira de Pesquisa Agropecuária (EMBRAPA), através do Programa Melhoramento de Amendoim, recentemente incluiu três novas cultivares de amendoim no Registro Nacional de Cultivares, sendo: a BRS 421 OL, a BRS 423 OL (SUASSUNA et al., 2020) e a BRS 425 OL (SUASSUNA et al., 2019). Essas cultivares apresentam como características o porte rasteiro, serem alto oleicas e produzirem grãos do tipo runner, visando atenderos mercados internos e externo de grãos. Na safra 2017/18, as cultivares BRS $421 \mathrm{OL}\left(6.711,1 \mathrm{~kg} \mathrm{ha}^{-1}\right)$, BRS $423 \mathrm{OL}\left(5.947,9 \mathrm{~kg} \mathrm{ha}^{-1}\right) \mathrm{e}$ BRS $425 \mathrm{OL}\left(6.407,4 \mathrm{~kg} \mathrm{ha}^{-1}\right)$ tiveram bom desempenho em Campo VerdeMT, evidenciando a possibilidade de cultivo do amendoim no cerrado brasileiro (SANTIN et al., 2019).

$O$ presente trabalho teve como objetivo avaliar o desempenho agronômico de genótipos de amendoim: três cultivares e duas linhagens avançadas desenvolvidas pelo Programa de Melhoramento do Amendoim da Embrapa, nas condições de Campo Verde-MT.

\section{Material e métodos}

O experimento foi desenvolvido no ano agrícola 2019/20 com semeadura manual no dia 04/11/2019 na área experimental pertencente ao Instituto Federal de Educação, Ciência e Tecnologia de Mato Grosso Campus São Vicente, Centro de Referência de Campo Verde, localizado no município de Campo Verde - MT, classificado como clima Aw segundo Koppen-Geiger.

O delineamento experimental utilizado foi em blocos casualizados, com quatro repetições. Os tratamentos constaram de cinco genótipos de amendoim rasteiro, três cultivares: BRS $421 \mathrm{OL}, \mathrm{BRS} 423 \mathrm{OL}, \mathrm{BRS} 425 \mathrm{OL}$ e duas linhagens avançadas: 17-1253 OL e 18-1952 OL, desenvolvidas pelo Programa de Melhoramento do Amendoim da Embrapa. As parcelas eram compostas por duas linhas de três metros de comprimento, espaçamento 
ente linhas de 0,90 m, intervalo entre parcelas de dois metros. A área total da parcela era de $5,4 \mathrm{~m}^{2}$ e foi considerada como parcela útil as linhas centrais com área de $3,6 \mathrm{~m}^{2}$.

Com base na necessidade do solo, foi realizado adubação de semeadura de $400 \mathrm{~kg} \mathrm{ha}^{-1}$ de superfosfato simples (SFS) no sulco de plantio. No tratamento de sementes foi utilizado carbendazim, na dose de $0,1 \mathrm{~L}$ por $100 \mathrm{~kg}$ de sementes. Foram feitas duas aplicações de adubação de cobertura, nos dias 14/12/2019 e 23/12/2019, ambas na dose de $50 \mathrm{~kg} \mathrm{ha}^{-1}$ de cloreto de potássio $(\mathrm{KCl})$ e uma aplicação de gesso agrícola no dia 16/12/2019 na dose de $500 \mathrm{~kg} \mathrm{ha}^{-1}$.

Os manejos cultural e fitossanitário foram realizados de acordo com as recomendações para a cultura. $O$ estande médio estabelecido foi de 16 plantas por metro linear. Aos 135 dias após a emergência (DAE), no final do ciclo dos genótipos, foi realizada a colheita manual. Foram realizadas as avaliações de massa de 100 grãos $(\mathrm{g})$ e produtividade de vagens $\left(\mathrm{kg} \mathrm{ha}^{-1} \mathrm{e}\right.$ sacas alqueire ${ }^{-1}$ ) quantificadas por meio de pesagem de vagens e grãos, da área central de cada parcela $\left(3,6 \mathrm{~m}^{2}\right)$. Também foi avaliada a severidade de mancha preta (Cercosporidium personatum) utilizando a escala diagramática da incidência com notas de 1 a 9 ao final do ciclo dos genótipos (SUBRAHMANYAM et al., 1982).

Os dados obtidos foram submetidos à análise de variância (Teste F) e as médias dos tratamentos foram comparados pelo teste de Scott-Knott a $5 \%$ de probabilidade, por meio do programa computacional SISVAR 5.6 (FERREIRA, 2019).

\section{Resultados e discussão}

As cultivares e linhagens avançadas de amendoim apresentaram diferença significativa para severidade de mancha preta (nota), massa de 100 grãos (g) e produtividade de vagens ( $\mathrm{kg} \mathrm{ha}^{-1}$ e sacas alqueire ${ }^{-1}$ ) (Tabela 1). 
Tabela 1. Severidade de mancha preta (nota), massa de 100 grãos (g) e produtividade de vagens $\left(\mathrm{kg} \mathrm{ha}^{-1}\right.$ e sacas alqueire ${ }^{-1}$ ) em função de diferentes genótipos de amendoim nas condições de Campo Verde-MT, safra 2019/20.

\begin{tabular}{|c|c|c|c|c|}
\hline \multirow{2}{*}{ Genótipos } & \multirow{2}{*}{$\begin{array}{c}\text { Severidade de } \\
\text { mancha preta (nota) }\end{array}$} & \multirow{2}{*}{$\begin{array}{l}\text { Massa de } \\
100 \text { grãos (g) }\end{array}$} & \multicolumn{2}{|c|}{ Produtividade de vagens } \\
\hline & & & $\left(\mathrm{kg} \mathrm{ha}^{-1}\right)$ & (sacas alqueire $^{-1}$ ) \\
\hline BRS 425 OL & $5,3 \mathrm{~b}$ & $69,7 \mathrm{~b}$ & $5.836,8 \mathrm{a}$ & 565,0 \\
\hline BRS $421 \mathrm{OL}$ & $5,0 \mathrm{~b}$ & $81,4 \mathrm{a}$ & $5.668,6 \mathrm{a}$ & 548,7 \\
\hline $17-1253 \mathrm{OL}$ & $5,8 \mathrm{a}$ & $72,9 \mathrm{~b}$ & $5.592,5 \mathrm{a}$ & 541,3 \\
\hline BRS 423 OL & $5,4 \mathrm{~b}$ & $70,9 \mathrm{~b}$ & $5.432,0 \mathrm{a}$ & 525,8 \\
\hline 18-1952 OL & $6,4 \mathrm{a}$ & $73,0 \mathrm{~b}$ & $4.935,6 \mathrm{~b}$ & 477,7 \\
\hline Média & 5,6 & 73,6 & $5.493,1$ & 531,7 \\
\hline C.V. (\%) & 9,5 & 5,0 & 4,1 & - \\
\hline $\mathrm{Pr}>\mathrm{Fc}$ & $0,0270^{*}$ & $0,0054^{*}$ & $0,0010^{*}$ & - \\
\hline
\end{tabular}

Sobre a severidade de mancha preta, as linhagens 18-1952 OL e 17-1253 OL apresentaram as maiores notas, com notas 6,4 e 5,8 respectivamente, evidenciando desfolha mais intensa do terço inferior, iniciando no terço médio das plantas, ao final do ciclo. Por outro lado, as cultivares BRS $421 \mathrm{OL}$, BRS $425 \mathrm{OL}$ e BRS $423 \mathrm{OL}$ apresentaram as menores notas, com 5,0; 5,3 e 5,4 respectivamente, com sintomas no terço médio das plantas, contudo sem desfolha. Ribeiro et al. (2019), em estudo de avaliação de severidade de mancha preta, realizados em Tupã-SP, obtiveram notas iguais estatisticamente para as linhagens sendo nota 7,3 para 18-1952 OL e 7,6 para 17-1253 OL.

As diferenças na massa de 100 grãos (g) resultaram em grãos de granulometrias diferentes, com diferença de $11,7 \mathrm{~g}$ entre as cultivares BRS $421 \mathrm{OL}(81,4 \mathrm{~g})$ e BRS $425 \mathrm{OL}(69,7 \mathrm{~g})$. A maior massa foi obtida com a cultivar BRS $421 \mathrm{OL}$, diferindo-se das demais. No entanto, as massas das linhagens 18-1952 OL (73,0 g), 17-1253 OL (72,9 g) e as cultivares BRS 423 OL (70,9 g) e BRS 425 OL (69,7 g) não diferiram entre si. Santin et al. (2019) e Rizzi et al. (2019) observaram maior massa de grãos na cultivar BRS 421 OL quando comparada a BRS 425 OL e BRS 423 OL, com ambos estudos realizados no estado do Mato Grosso. 
As maiores produtividades foram obtidas com os genótipos BRS 425 OL (5.836,8 kg ha-1), BRS $421 \mathrm{OL}\left(5.668,6 \mathrm{~kg} \mathrm{ha}^{-1}\right), 17-1253 \mathrm{OL}(5.592,5 \mathrm{~kg}$ $\left.\mathrm{ha}^{-1}\right)$ e BRS $423 \mathrm{OL}\left(5.432,0 \mathrm{~kg} \mathrm{ha}^{-1}\right)$. Santin et al. (2019), também obtiveram igualdade estatística e maiores produtividades, com as cultivares BRS 421 OL, BRS 425 e BRS 423 OL, nas condições de Campo Verde-MT, demonstrando a possível adaptação agronômica. Suassuna et al. (2018), observaram que as produtividades das cultivares BRS $425 \mathrm{OL}$, BRS $423 \mathrm{OL}$ e BRS 421 OL, não diferiram entre si, em estudo realizado nas condições de Primavera do Leste-MT.

A cultivar que obteve maior produtividade (BRS $425 \mathrm{OL}$ ) apresentou menor massa de 100 grãos. A linhagem 18-1952 OL $\left(5.493,6 \mathrm{~kg} \mathrm{ha}^{-1}\right)$ foi a menos produtiva, com redução de 12 e 15\% quando comparada a 17-1253 OL e BRS $425 \mathrm{OL}$, respectivamente. Martins et al. (2019) observaram a linhagem 18-1952 OL com produtividade 9 e 19\% inferiores às obtidas no 17-1253 OL e BRS 425 OL, respectivamente.

\section{Conclusões}

Todas as cultivares apresentaram as menores notas de severidade de mancha preta. Os genótipos BRS 425 OL, BRS 421 OL, 17-1253 OL e BRS $423 \mathrm{OL}$ foram os mais produtivos. Constata-se nesta safra resultados de adaptação agronômica das cultivares BRS 421 OL, BRS 423 OL e BRS $425 \mathrm{OL}$ nas condições edafoclimáticas presentes durante o experimento.

\section{Agradecimentos}

Os autores agradecem ao IFMT Campus São Vicente - Centro de Referência de Campo Verde e ao Programa de Melhoramento do Amendoim da Embrapa, vinculado ao projeto SEG 20.18.01.021.00, localizada em Santo Antônio do Goiás-GO, pelo apoio. 


\section{Referências}

BERTIOLI, D. J.; SEIJO, G.; FREITAS, F. O.; VALLS, J. F. M.; BERTIOLI, S. C. M. L.; MORETZSOHN, M. C. An overview of peanut and its wild relatives. Plant Genetic Resources: characterization and utilization, v. 9, n. 1, p. 134-149, 2011.

CONAB. Acompanhamento da Safra Brasileira de grãos. Safra 2019/20 Sétimo levantamento, v. 7, n. 7, p. 1-25, abr. 2020. Disponível em: <https://www.conab.gov.br/info-agro/safras >. Acesso em: 16 maio 2020.

FERREIRA, D. F. SISVAR: a computer analysis system to fixed effects split plot type designs. Revista Brasileira de Biometria, [S.L.], v. 37, n. 4, p. 529, 20 dez. 2019. http://dx.doi.org/10.28951/rbb.v37i4.450

JUDD, W. S.; CAMPBELL, C. S.; KELLOGG, E. A.; STEVENS, P. F. Plant systematics: a phylogenetic approach. Massachusetts: Sinauer Associates, 1999. 464p.

MARTINS, K. B. B.; RODRIGUES, L. L.; HEUERT, J.; XAVIER, M. F. N.; SUASSUANA, T. M. F.; BETIOL, R. A. B. Desempenho agronômico de novas linhagens de amendoim no Cerrado. In: Anais do Encontro Sobre a Cultura do Amendoim, 16., 2019, Jaboticabal. Anais eletrônicos... Campinas: GALOÁ, 2019. Disponível em: $<$ https://proceedings.science/encontro-amendoim-2019/papers/desempenhoagronomico-de-novas-linhagens-de-amendoim-no-cerrado>. Acesso em: 17 maio 2020.

RIBEIRO, R. P.; HEUERT, J.; SOAVE, J. H.; SANTOS, L. C. C.; SUASSUANA, T. M. F. Avaliação de severidade de mancha preta e mancha anelar em diferentes genótipos de amendoim na região da Alta Paulista. In: Anais do Encontro Sobre a Cultura do Amendoim, 16., 2019, Jaboticabal. Anais eletrônicos... Campinas: GALOÁ, 2019. Disponível em: $<$ https://proceedings.science/encontro-amendoim-2019/papers/avaliacao-deseveridade-de-mancha-preta-e-mancha-anelar-em-diferentes-genotipos-deamendoim-na-regiao-da-alta-paulista>. Acesso em: 17 maio 2020.

RIZZI, T. S.; OLIBONE, D.; LODEA, L.; HEUERT, J.; XAVIER, M. F. N.; SUASSUNA, T. M F. Desempenho de cultivares de amendoim na região Médio-Norte Mato-grossense. In: Anais do Encontro Sobre a Cultura do Amendoim, 16., 2019, Jaboticabal. Anais eletrônicos... Campinas: GALOÁ, 2019. Disponível em: <https://proceedings.science/encontro-amendoim2019/papers/desempenho-de-cultivares-de-amendoim-na-regiao-medionorte-mato-grossense>. Acesso em: 17 maio 2020.

SANTIN, V.; PEROZINI, A. C.; ARAÚJO, C.; GIRON, F. G.; HEUERT, J.; XAVIER, M. F. N.; SUASSUNA, T. M. F. Desempenho de cultivares de amendoim nas condições de Campo Verde-MT. In: Anais do Encontro Sobre a Cultura do Amendoim, 16., 2019, Jaboticabal. Anais eletrônicos... Campinas: GALOÁ, 2019. Disponível em: 
$<$ https://proceedings.science/encontro-amendoim-2019/papers/desempenhode-cultivares-de-amendoim-nas-condicoes-de-campo-verde-mt>. Acesso em: 16 maio 2020.

SUASSUNA, T. M. F. et al. 'BRS 421' and 'BRS 423': high oleic peanut cultivars for production in Brazil. Crop Breeding Applied Biotechnology, $v$. 20, n. 1, e28932018, 2020.

SUASSUNA, T. M. F. et al. BRS 425: the first runner peanut cultivar related to wild ancestral species. Crop Breeding Applied Biotechnology, v. 19, n. 3, p. 373-377, 2019.

SUASSUNA, T. M. F.; HEUERT, J.; BOGGIANI, J. C.; PERINA, F. J.; SOFIATTI, V.; BETTINI, P. C.; OLIVEIRA, M. C. T.; LEONEL, C. L. Desempenho de linhagens de amendoim na região do Cerrado. In: Anais do Encontro Sobre a Cultura do Amendoim, 15., 2018, Jaboticabal. Anais eletrônicos... Campinas: GALOÁ, 2018. Disponível em: $<$ https://proceedings.science/encontro-amendoim-2018/papers/desempenhode-linhagens-de-amendoim-na-regiao-do-cerrado>. Acesso em: 25 maio 2020.

SUBRAHMANYAM, P.; MCDONALD, D.; GIBBONS, R. W.; NIGAM, S. N.; NEVILL, D. J. Resistance to rust and late leaf spot diseases in some genotypes of Arachis hypogaea. Peanut Science, v. 9, p. 9-14, 1982. 